\title{
Thermophilic Bacteria Strictly Obey Szybalski's Transcription Direction Rule and Politely Purine-Load RNAs with Both Adenine and Guanine
}

\author{
Perry J. Lao and Donald R. Forsdyke ${ }^{1}$ \\ Department of Biochemistry, Queen's University, Kingston, Ontario, K7L 3N6, Canada
}

\begin{abstract}
When transcription is to the right of the promoter, the "top," mRNA-synonymous strand of DNA tends to be purine-rich. When transcription is to the left of the promoter, the top, mRNA-template strand tends to be pyrimidine-rich. This transcription-direction rule suggests that there has been an evolutionary selection pressure for the purine-loading of RNAs. The politeness hypothesis states that purine-loading prevents distracting RNA-RNA interactions and excessive formation of double-stranded RNA, which might trigger various intracellular alarms. Because RNA-RNA interactions have a distinct entropy-driven component, the pressure for the evolution of purine-loading might be greater in organisms living at high temperatures. In support of this, we find that Chargaff differences (a measure of purine-loading) are greater in thermophiles than in nonthermophiles and extend to both purine bases. In thermophiles the pressure to purine-load affects codon choice, indicating that some features of their amino acid composition (e.g., high levels of glutamic acid) might reflect purine-loading pressure (i.e., constraints on $\mathrm{mRNA}$ ) rather than direct constraints on protein structure and function.
\end{abstract}

Duplex DNA can be represented as two horizontal lines, representing "top" $\left(5^{\prime} \rightarrow 3^{\prime}\right)$ and "bottom" $\left(3^{\prime} \rightarrow 5^{\prime}\right)$ strands. When transcription is to the right of the promoter, the top, mRNA-synonymous strand tends to be purine-rich. When transcription is to the left of the promoter, the top, mRNA-template strand tends to be pyrimidine-rich (Szybalski et al. 1966; Smithies et al. 1981). It follows that mRNAs, whatever the direction of their transcription, tend to be purinerich (Bell et al. 1998; Dang et al. 1998). Usually one of the two purine bases is most heavily involved in purine-loading, and the other may appear indifferent. For organisms of low genomic $(\mathrm{C}+\mathrm{G}) \%$ the purine is usually A. For organisms of high $(\mathrm{C}+\mathrm{G}) \%$ the purine is usually G. The extra purines are located in the loop regions of computer-folded mRNA structures (Bell and Forsdyke 1999a,b).

To explain the phenomenon of purine-loading, it was pointed out that the physical and chemical state of the "crowded" cytosol (Fulton 1982; Forsdyke 1995) is probably adapted to facilitate a reaction of fundamental importance-tRNA-mRNA interaction. This connection between genotype and phenotype (mRNA translation) must occur rapidly and with high specificity. If cytosolic conditions were such as to optimize this process, then there would be an increased prob-

'Corresponding author.

E-MAIL forsdyke@post.queensu.ca; FAX (613) 533-2497. ability not only of efficient tRNA-mRNA interactions, but also of efficient mRNA-mRNA interactions. These would initiate by way of "kissing" between the loops of folded RNAs (Eguchi et al. 1991). Such interactions might (1) directly impede protein synthesis, and (2) generate double-stranded RNA segments of lengths sufficient to trigger various intracellular alarms (Cristillo 1998; Fire 1999; Forsdyke 1999a; A.D. Cristillo, T.P. Lillicrap, and D.R. Forsdyke, unpubl.). Thus, there would have been a selection pressure for mRNAs to be "polite" (Zuckerkandl 1986) and avoid unnecessary interactions. This would have been achieved, wherever compatible with other mRNA functions, by loading loops of all mRNAs with non-Watson-Crick pairing bases (e.g., all purines or all pyrimidines).

Exploratory kissing interactions between hybridizing nucleic acids involve transient stacking interactions (Eguchi et al. 1991), with the exclusion of structured water. Such reactions have a strong entropydriven component (Cantor and Schimmel 1980) and so might increase as temperature increases (Lauffer 1975). Hence, perhaps counterintuitively, nucleic acids should be more "sticky" at high temperatures and the selection pressure to avoid formation of doublestranded RNA should be greater. To examine this, we compare the magnitude (evaluated as Chargaff differences) and range (one or both purines) of purineloading in the genomes of thermophilic bacteria with those of the genomes of mesophilic bacteria, which 
normally exist at $37^{\circ} \mathrm{C}$ or lower temperatures. We also examine the extent to which the pressure to purineload has affected codon choice, and hence, potentially, protein composition and function.

\section{RESULTS}

\section{Large Chargaff Differences in Methanococcus jannaschii}

Figure 1 shows a plot of Chargaff differences (\%) along a segment of the genome of the thermophilic bacterium Methanococcus jannaschii. In previous studies of nonthermophilic organisms, such plots had to be carefully examined to see whether the $\mathrm{S}$ or the $\mathrm{W}$ bases were the best predictors of transcription direction in accordance with Szybalski's transcription direction rule. Chargaff differences were seldom $>20 \%$ (especially Chargaff differences for the $\mathrm{S}$ bases), and simply plotting the ratio of purines to pyrimidines (R/Y) was not particularly informative (Bell et al. 1998; Dang et al. 1998; Bell and Forsdyke 1999a,b). In contrast, for $M$. jannaschii it is observed that (1) both purines follow Szybalski's transcription direction rule, (2) the magnitude of the Chargaff differences is often $>20 \%$ (especially Chargaff differences for the $S$ bases), and (3) the $\mathrm{R} / \mathrm{Y}$ ratio strongly correlates with transcription direction.

Even a small rightward-transcribed ORF (in the 15.5 - to 15.8 -kb region), which is surrounded by leftward-transcribed ORFs, is detectable as a small dip in the S-base plot $(\mathrm{G}>\mathrm{C})$, although the $\mathrm{W}$-base plot is not affected. No ORF has been reported at the beginning of the sequence (1- to $2-\mathrm{kb}$ region), but the curve pattern $(A>T ; G>C)$ predicts that any ORFs found here should be transcribed to the right of the promoter.

\section{Quadrant Analysis of M. jannaschii}

To show that these features of a 20-kb segment (Fig. 1) are typical of the whole genome, the two Chargaff differences (for the $\mathrm{W}$ and $\mathrm{S}$ bases) were plotted against each other to generate quadrant plots (Bell et al. 1998) for leftward-transcribed ORFs (Fig. 2a) and for rightward-transcribed ORFs (Fig. 2b). Each point in such plots represents a $1-\mathrm{kb}$ window. Because $M$. jannaschii is $1.66 \mathrm{Mb}$ and windows are taken at $0.1-\mathrm{kb}$ intervals, there are several thousand points in each plot. For windows whose centers overlap leftward-transcribed ORFs, most points indicate enrichment both in $\mathrm{T}$ and $\mathrm{C}$, implying that the corresponding mRNA synonymous strands would be enriched both in A and G. For windows whose centers overlap rightward-transcribed ORFs, most points indicate enrichment both in A and $G$, again implying that the mRNA synonymous strands are enriched both in A and G.

As in Figure 1, Chargaff differences for the $S$ bases are generally greater than those for the $\mathrm{W}$ bases (e.g., in Fig. $2 b$ points are generally farther to the left of the ordinate, indicating G-richness, than they are above the abscissa, indicating A-richness). Although widely scattered, the points fit linear regressions sloping downward, so that, as suggested by Figure 1, it is likely that windows enriched in one pyrimidine are also enriched in the other (Fig. 2a) and that windows enriched in one purine are also enriched in the other (Fig. 2b).

\section{Large Chargaff Differences in Other Thermophiles}

The extreme purine-loading found for the entire genome of M. jannaschii was also found for large segments of the genomes of two other thermophilic bacteria (Table 1). The thermophilic bacteria strictly comply with Szybalski's transcription direction rule for both WatsonCrick base pairs, and the magnitude of the differences is generally greater than in the case of the nonthermophilic bacteria. The latter sometimes do not comply with respect to one Watson-Crick base pair (for the Escherichia coli segment,
Figure 1 Variation of Chargaff differences (\%) and purine/pyrimidine (R/Y) ratios along the first $20 \mathrm{~kb}$ of the genomic sequence of the thermophile $M$. jannaschii. A 1-kb sequence window was moved in steps of $0.1 \mathrm{~kb}$, and base compositions were determined in each window. From these, Chargaff differences ( $O$ for the $W$ bases; $\square$ for the $S$ bases), and $R / Y$ ratios (continuous putative ORFs are shown as boxes (open for transcription to the left; shaded for transcription to the right; orientations are also marked by horizontal arrows below each box). Although most ORFs correspond to hypothetical proteins, some tentatively identified ORFs have been labeled: (aspB1) Aspartate aminotransferase; (activ) activator of 2-hydroxyglutaryl-CoA dehydratase; (formate dehyd.) $\alpha$ and $\beta$ subunits of formate dehydrogenase; (glutar) $\beta$ subunit of 2 -hydroxyglutaryl-CoA dehydratase; (pyruvate decarb.) phosphonopyruvate decarboxylase; transposase; (gatB) Asp-tRNA amidotransferase, subunit B.
Genome Research www.genome.org 
(a) Leftward-Transcribed ORFs

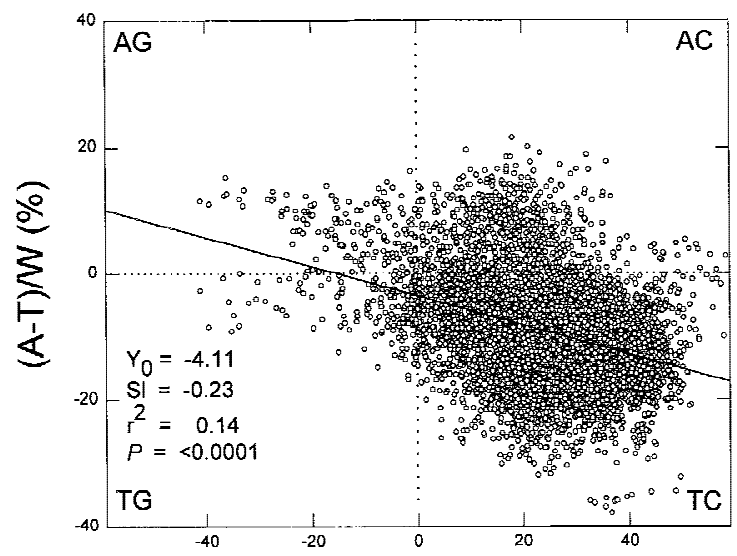

(b) Rightward-Transcribed ORFs

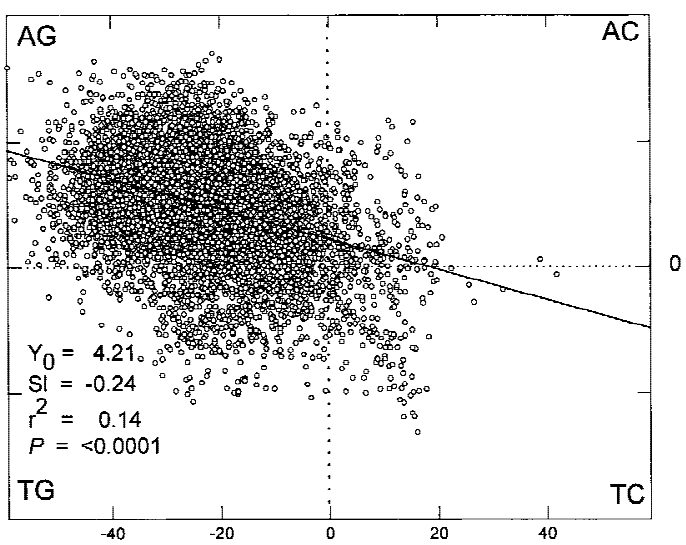

$(\mathrm{C}-\mathrm{G}) / \mathrm{S} \%$

Figure 2 Quadrant analysis of Chargaff differences (\%) for the W and $\mathrm{S}$ bases in 1-kb windows from the top strand of the $M$. jannaschii genome. Each quadrant corresponds to windows enriched for two particular bases, as indicated at the corners. (a) The 7494 windows whose centers overlap leftward-transcribed ORFs; $(b)$ the 8202 windows whose centers overlap rightward-transcribed ORFs. The diagonal lines are the least squares regression lines. Listed are values for intercepts at the ordinate $\left(Y_{0}\right)$, slopes $(\mathrm{SI})$, squares of correlation coefficients $\left(r^{2}\right)$, and probabilities that slopes are not significantly different from zero $(P)$. Similar values were obtained for plots using every tenth window to avoid window overlap (e.g., $P<0.0001$ ).

rightward-transcribed ORFs are slightly T-rich; for the Haemophilus influenzae segment, leftward transcribed ORFs are slightly A-rich).

The difference between thermophiles and nonthermophiles was more readily appreciated when the four Chargaff differences values (base excesses) for each organism were combined to provide an index of the purine-loading of the corresponding RNAs. For the thermophiles the index is simply the sum of the four absolute Chargaff differences, as the transcription rule is followed in each of the four cases. For the nonthermophiles, in cases where the transcription rule was not followed, the corresponding values were subtracted from the overall sum. On average, the three thermophiles show more purine-loading than the three nonthermophiles (Table 1). Purine-loading in thermophiles $[(153+208+288) /(3)]$ exceeds that of nonthermophiles $[(42+64+64) /(3)]$ by 160 bases/kb window $(P=0.04$; paired $t$-test with $2 \mathrm{df}$ ).

\section{More Purine-Loading at Low $(\mathrm{C}+\mathrm{G})$}

For thermophiles the purine-loading index shows an inverse linear relationship to $(\mathrm{C}+\mathrm{G}) \%$, with a slope that is significantly different from zero $\left(r^{2}=0.994\right.$; $P=0.05$ ). The relationship reflects purine-loading with $\mathrm{G}$ residues more than with $\mathrm{A}$ residues [i.e., with decreasing $(C+G) \%$ there is an increasing tendency for $\mathrm{C}$-excess in leftward-transcribed ORFs and for G-excess in rightward-transcribed ORFs]. Thus, although there are fewer $\mathrm{S}$ bases available to support these excesses when the $(\mathrm{C}+\mathrm{G}) \%$ is low, those present are more readily utilized for the purine-loading function (i.e., they are likely to be locally unpaired in loops, rather than in stems). Whereas in their absolute numbers, the $\mathrm{S}$ bases and the $\mathrm{W}$ bases contribute about equally to purine-loading in thermophilic bacteria, usually one class dominates in the nonthermophilic bacteria.

\section{Genome-Wide Distribution of Purine-Loading}

That large segments of genomes are likely to be representative of the entire genomes with respect to purineloading was shown for M. jannaschii. The genome was divided into six segments of approx. $276.5 \mathrm{~kb}$ each. At this level of resolution, there are only minor fluctuations between segments in $(\mathrm{C}+\mathrm{G}) \%$, Chargaff differences, and purine-loading indices (Table 2). For example, for rightward-transcribed ORFs, the genomic average Chargaff difference (\%) for the $\mathrm{W}$ bases is $9.92 \pm 0.09$ (i.e., $\mathrm{A}>\mathrm{T}$; Table 1), and corresponding values for the six segments range from 8.46 to 10.57 .

The genome of $M$. jannaschii shows a remarkable symmetry between leftward and rightward ORFs in the actual numbers of $\mathrm{S}$ and $\mathrm{W}$ bases contributing to purine-loading (Table 1 ). Thus, the average $1-\mathrm{kb}$ window has pyrimidine excesses of 68 (T) and 76 (C) for leftward-transcribed ORFs, and purine excesses of 68 (A) and $76(\mathrm{G})$ for rightward-transcribed ORFs. This tendency toward symmetry also occurs in the six segments (Table 2) and is precise in segment 2.

\section{Influence of the Origin of Replication?}

Although leftward and rightward ORFs are covered by approximately equal numbers of overlapping $1-\mathrm{kb}$ windows (7494 and 8202, respectively), the distribu- 


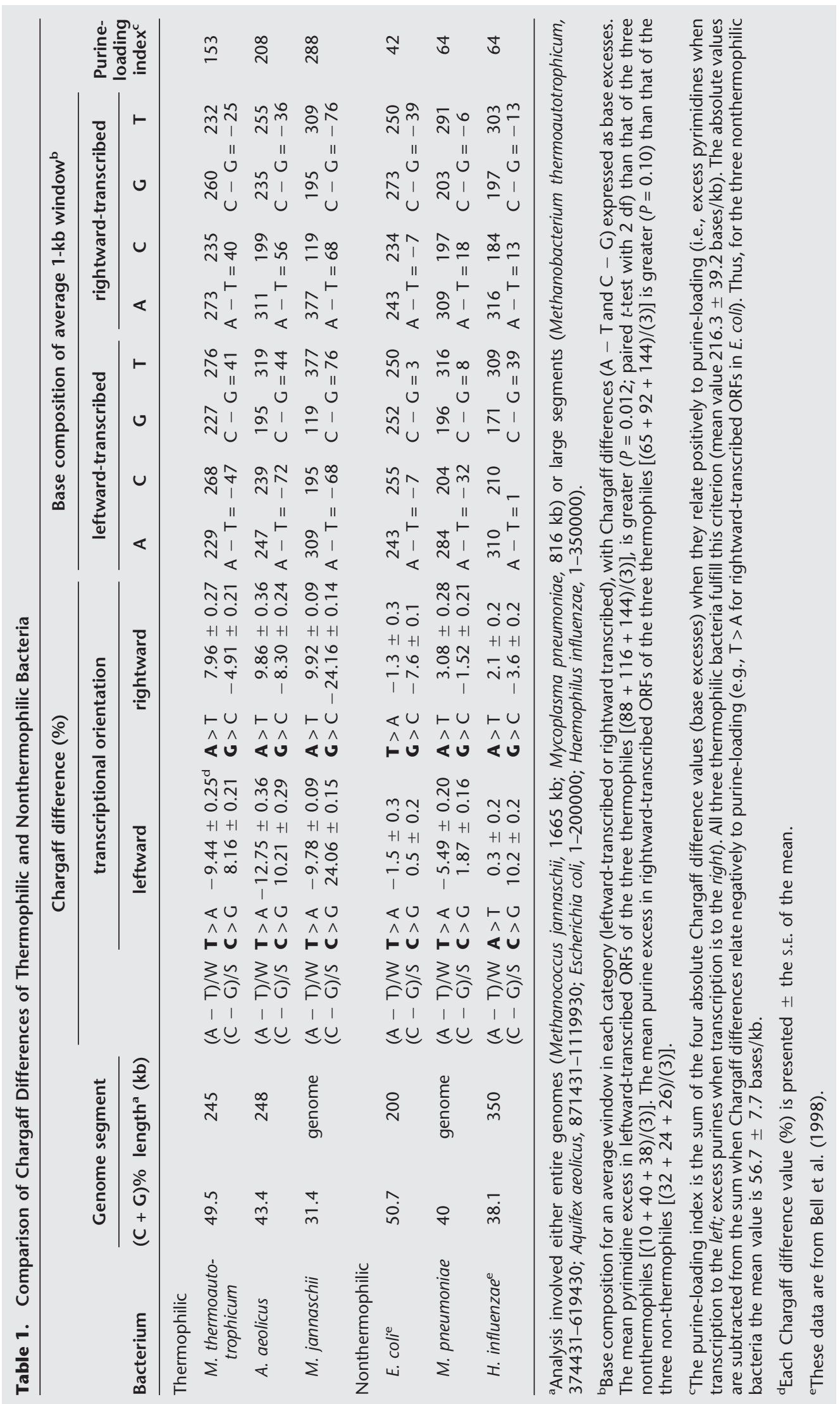




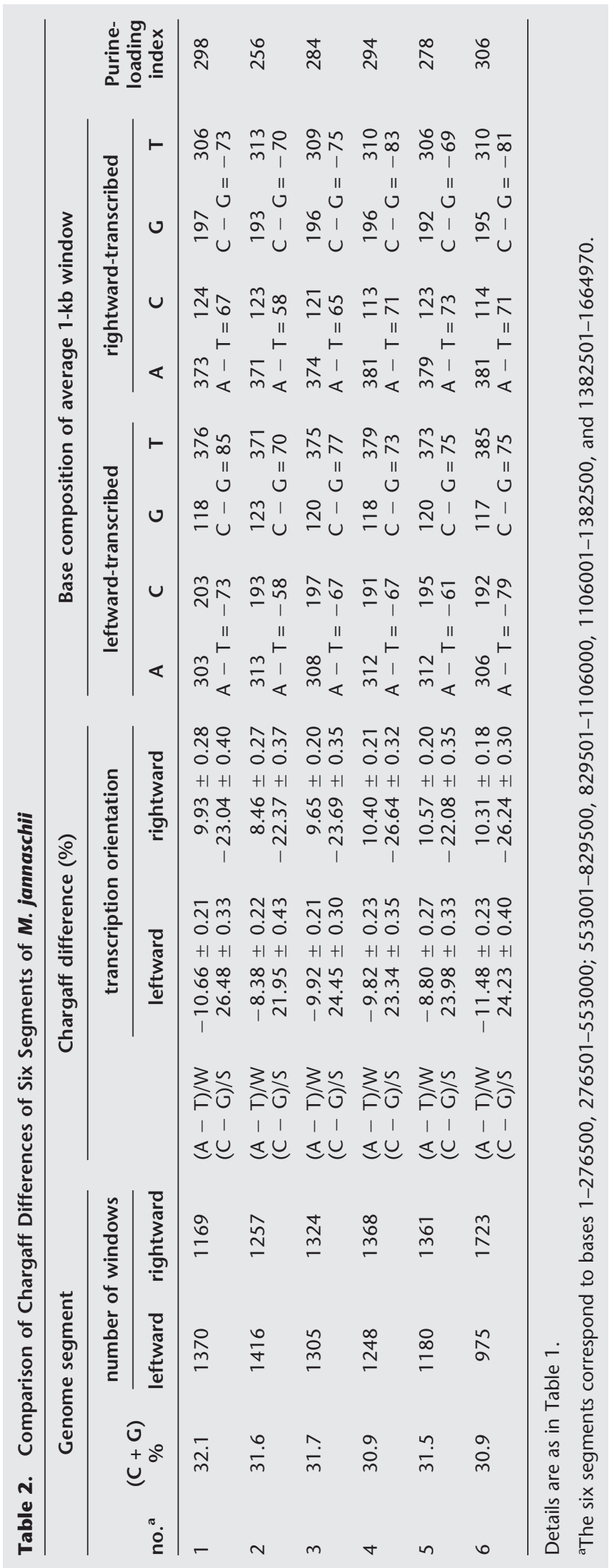


tion varies (Table 2). The number of windows corresponding to leftward ORFs tends to decrease with segment number, whereas the number of windows corresponding to rightward ORFs tends to increase with segment number. Because the M. jannaschii chromosome is circular, a sharp switch is indicated between segments 6 and 1 from a predominantly purine-rich top strand (reflecting an excess of windows covering rightward ORFs in segment 6) to a predominantly pyrimidine-rich top strand (reflecting an excess of windows covering leftward ORFs in segment 1). This probably relates to the origin of replication (site not currently known), as when windows much greater than 1 $\mathrm{kb}$ are used for Chargaff difference analysis (thus tending to obscure the local effects of individual ORFs), the results can provide a guide to the position of the origin of replication (Smithies et al. 1981; Lobry 1996).

\section{Same Optimum Window for Thermophiles and Nonthermophiles}

In previous studies the optimum window $(1 \mathrm{~kb})$ for examining Chargaff differences was determined in a range of organisms by comparing natural with the cor-

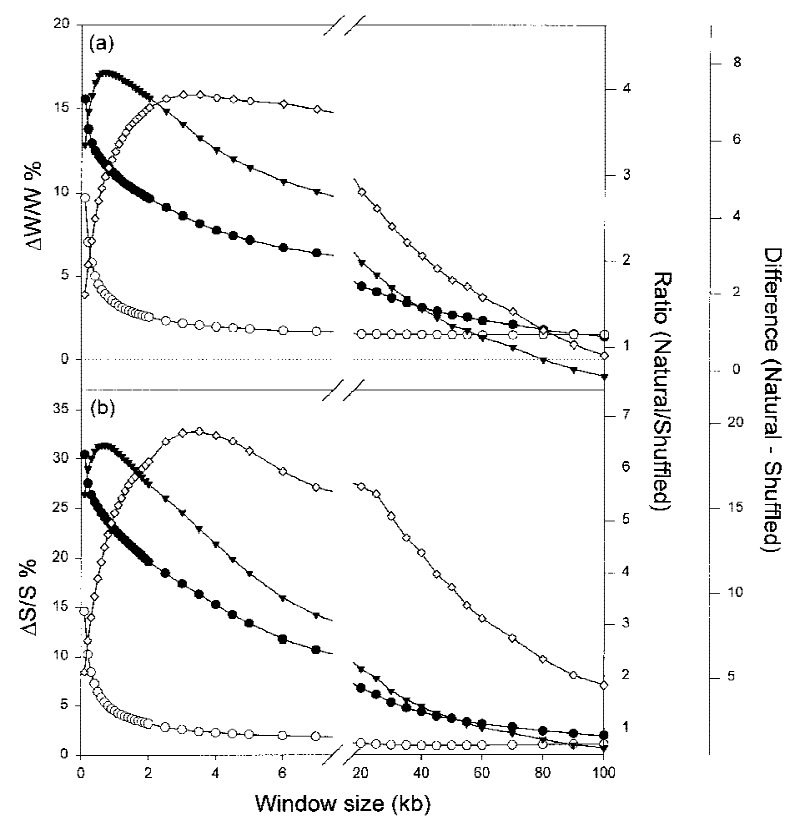

Figure 3 Variation of average Chargaff difference values with size of windows in $M$. jannaschii. Windows of varying size were moved along the first $276.5 \mathrm{~kb}$ of the genome in steps of 100 nucleotides, and base compositions were determined in each window. Average absolute Chargaff differences (\%) for each window size are plotted either as (natural sequence), or $\bigcirc$ (shuffled sequence). ( $\boldsymbol{\Delta})$ The difference between these values (the average Chargaff difference for the natural sequence less the average Chargaff difference for the shuffled sequence). ( $\diamond)$ The ratio of these values. The total number of windows of a given size used to calculate average Chargaff differences varied with sequence length. Thus, in a 100-kb sequence there would be 999 windows of $0.2 \mathrm{~kb}$ and one window of $100 \mathrm{~kb}$. responding shuffled sequences (Bell et al. 1998; Dang et al. 1998; Bell and Forsdyke 1999a). A similar study of the three thermophile genomes considered above indicated that a 1-kb window would also be optimum in these organisms. For example, for Figure 3 absolute Chargaff difference values were plotted against window size in a $276.5-\mathrm{kb}$ segment of $M$. jannaschii. The difference between values of points on the curves for the natural and shuffled sequences reaches an optimum at a window size of $\sim 1 \mathrm{~kb}$. As with the nonthermophiles, the ratio of the values on these curves reaches an optimum at higher window sizes. It was concluded that it was appropriate to use $1-\mathrm{kb}$ windows when comparing the Chargaff differences of thermophile and nonthermophile genomes.

\section{Purine-Loading Affects Codon Choice}

The strong pressure on thermophiles to purine-load their mRNAs, as revealed by Chargaff difference analysis, suggested that choice of synonymous codons might provide an independent measure of this evolutionary force. Furthermore, thermophiles show some regularities in amino acid compositions (Kagawa et al. 1984; Deckert et al. 1998; Jaenicke and Bohm 1998), raising the possibility that the choice of nonsynonymous codons might also be affected.

Table 3 shows data for some amino acids corresponding to purine-rich codons. In the case of glycine (4 codons) and arginine (6 codons) the opportunity is provided for choice of synonymous codon, and in both cases purine-rich codons are preferred by thermophiles. Thus, thermophiles prefer GGR over GGY, and nonthermophiles prefer GGY over GGR. This suggests a selection pressure acting at the nucleic acid level, rather than at the protein level.

There is a significant increase in codons for glutamic acid in thermophiles, consistent with a selection pressure on nonsynonymous codons. However, although there is a suggestion of a similar pressure for arginine, it is not found for aspartic acid or lysine. The thermophile Archaeoglobus fulgidus is very similar to the other thermophiles (Table 3), indicating that in future work, purine-loading might also be demonstrable by the Chargaff difference method in this organism.

\section{DISCUSSION}

\section{An Adaptation for Survival at High Temperatures}

It is likely that Szybalski's transcription direction rule is a consequence of purine-loading (Bell and Forsdyke 1999b). Under this assumption, the difference between thermophilic and nonthermophilic bacteria in (1) the extent of their compliance with Szybalski's transcription direction rule (Table 1), and (2) their choice of purine-rich codons (Table 3 ) suggests that adaptation 
Table 3. Comparison of Amino Acid Frequency and Codon Choice of Thermophilic and Nonthermophilic Bacteria

\begin{tabular}{|c|c|c|c|c|c|c|c|c|c|c|c|}
\hline \multirow{3}{*}{$\begin{array}{l}\text { Amino acid } \\
\text { codons } \\
\text { bacterium }\end{array}$} & \multirow[b]{3}{*}{$(C+G) \%$} & \multicolumn{10}{|c|}{ Specific codons/1000 codons } \\
\hline & & \multicolumn{3}{|c|}{ glycine } & \multirow{2}{*}{$\frac{\text { glutamic }}{\text { GAR }}$} & \multirow{2}{*}{$\frac{\text { aspartic }}{\text { GAY }}$} & \multirow{2}{*}{$\frac{\text { lysine }}{\text { AAR }}$} & \multicolumn{4}{|c|}{ arginine } \\
\hline & & GGR & GGY & total & & & & AGR & CGR & CGY & total \\
\hline \multicolumn{12}{|l|}{ Thermophilic } \\
\hline M. thermoautotrophicum & 49.5 & 38.2 & 41.4 & 79.6 & 81.5 & 59.1 & 45.6 & 53.3 & 5.8 & 8.4 & 67.4 \\
\hline A. fulgidus & 48.5 & 41.2 & 31 & 72.2 & 88.7 & 48.7 & 68.3 & 51.7 & 2.4 & 3.3 & 57.4 \\
\hline A. aeolicus & 43.4 & 43.5 & 23.6 & 67.1 & 96.2 & 43 & 94.1 & 44.9 & 1.3 & 2.7 & 49 \\
\hline M. jannaschii & 31.4 & 48.8 & 17.2 & 66.1 & 86.2 & 54.9 & 103.6 & 37.4 & 0.5 & 0.4 & 38.3 \\
\hline Mean & & 42.9 & 28.3 & 71.2 & 88.1 & 51.4 & 77.9 & 46.8 & 2.5 & 3.7 & 53 \\
\hline \multicolumn{12}{|l|}{ Nonthermophilic } \\
\hline E. coli & 50.7 & 20.5 & 52.5 & 73 & 56.9 & 51.4 & 45.5 & 5 & 10 & 40.8 & 55.7 \\
\hline M. pneumoniae & 40 & 15.3 & 39.6 & 54.9 & 56.8 & 49.4 & 85.2 & 6.8 & 7.5 & 20.5 & 34.9 \\
\hline H. influenzae & 38.1 & 18.4 & 48.2 & 66.6 & 63.8 & 49.8 & 63.8 & 4.6 & 6.6 & 33.3 & 44.4 \\
\hline M. genitalium & 32 & 18.2 & 27.9 & 46.1 & 56.6 & 49 & 94.6 & 18.7 & 2.4 & 9.9 & 31 \\
\hline Mean & & $\begin{array}{l}18.1 \\
0.003\end{array}$ & $\begin{array}{l}42 \\
0.03\end{array}$ & $\begin{array}{c}60.1 \\
0.09\end{array}$ & $\begin{array}{l}58.5 \\
0.0005\end{array}$ & $\begin{array}{c}49.9 \\
0.68\end{array}$ & $\begin{array}{c}72.3 \\
0.61\end{array}$ & $\begin{array}{l}8.8 \\
0.01\end{array}$ & $\begin{array}{l}6.6 \\
0.01\end{array}$ & $\begin{array}{c}26.1 \\
0.03\end{array}$ & $\begin{array}{c}41.5 \\
0.06\end{array}$ \\
\hline
\end{tabular}

for survival at high temperatures requires that mRNAs be more heavily purine-loaded. Consistent with this, in a survey of 12 chloroplast genomes (R.J. Rasile and D.R. Forsdyke, unpubl.) we find that those of the thermophile Cyanidium caldarium have a greater purineloading index (108 bases/kb) than those of 11 nonthermophilic organisms (average $41.1 \pm 6.2$ bases/kb). An extreme example of the latter is the genome of chloroplasts of the parasitic plant Epiphagus virginiana, in which the chloroplasts are degenerate so that pressure on mRNAs to be polite is likely to be decreased (purineloading index only 22 bases/kb).

\section{The Politeness Hypothesis}

The Gibb's free energy equation $(\Delta G=\Delta H-T \Delta S)$ implies that with increasing temperature, reactions with a significant entropy-driven component can occur more readily (Lauffer 1975). Because the base-pairing involved in RNA-RNA interactions has a considerable entropic component (Cantor and Schimmel 1980), such interactions, whether desirable or undesirable, should be favored at high temperatures. Thus, if chemically and biologically feasible, it is possible that RNA sequences would have adapted to avoid undesirable interactions while not impairing desirable ones. Purineloading would seem to achieve this. In general, mRNAs "drive" politely on the purine "side of the road," and thermophile mRNAs appear excessively polite. The politeness is not trivial (contrast the "polite DNA" of Zuckerkandl 1986). Driving on the correct side of the road is conducive to efficient highway operation. Failure to do so might be lethal if it led to the formation of dsRNA and the false triggering of intracellular alarms.

\section{Exceptions to Szybalski’s Rule}

There are exceptions to Szybalski's rule (Cristillo 1998; Bell and Forsdyke 1999b; A.D. Cristillo, T.P. Lillicrap, and D.R. Forsdyke, unpubl.). Certain viruses with a prolonged period of clinical latency load their mRNAs with pyrimidines. Thus, whereas human immunodeficiency virus 1 (moderately committed to latency) is polite (mRNAs heavily purine-loaded), human T-cell leukemia virus 1 (profoundly committed to latency) is extremely impolite (mRNAs heavily pyrimidineloaded). Epstein-Barr virus (like many other herpes viruses) is also profoundly committed to latency and pyrimidine-loads most of its RNAs. However, unlike human T cell leukemia virus 1, Epstein-Barr virus has an important transcript expressed in all forms of latency, the transcript encoding the EBNA-1 antigen. Remarkably, this transcript is polite, its purine-loading being amplified by inclusion of a simple-sequence element encoding, by preferential employment of purine-rich codons, a glycine-alanine repeat that can be removed without greatly affecting EBNA-1 function. Despite this, much current work is based on the premise that in the compact virus genome, the simple-sequence element persists because of a function at the protein level rather than at the nucleic acid level (for references, see Lee et al. 1999).

\section{Purine-Loading Might Affect the Composition of Proteins}

Conventional natural selection provides an extrinsic pressure on the phenotype and so determines which genotypes will survive. However, it has long been recognized that genomes are also molded by intrinsic 
forces (Romanes 1886; Forsdyke 1999b,c). One such force is a component of the base composition of DNA- $(\mathrm{C}+\mathrm{G}) \%$. This can affect protein composition and, hence, possibly the phenotype (Sueoka 1961; Ball 1973; Grantham 1980; Bronson and Anderson 1994; Forsdyke 1996, 1998). In light of the present work it appears that another component of base composition, $\mathrm{R} / \mathrm{Y}$, may also affect protein composition and, hence, possibly the phenotype.

We have identified purine-loading as an evolutionary force and have suggested an adaptive basis related to the intrinsic workings of the cell (Bell et al. 1998; Bell and Forsdyke 1999b; Fire 1999; Forsdyke 1999a). Thermophilic bacteria appear particularly susceptible to this force, so that the pressure to purineload RNA in these organisms might have been powerful enough to affect choice both of synonymous and nonsynonymous codons. In thermophiles, an increase in the proportion of glutamic acid (encoded by codons containing only purines) has been noted (Deckert et al. 1998), and this is confirmed in Table 3. Although it is tempting to believe that all such changes in the amino acid composition of proteins are related to the need to maintain protein stability and function at high temperatures (Kagawa et al. 1984; Jaenicke and Bohm 1998), our results raise the possibility that the needs of efficient mRNA function at high temperatures might also have affected the composition of proteins. However, Jaenicke and Bohm (1998) find it difficult to define what they call "traffic rules" of thermophilic adaptation in terms of significant differences in amino acid composition.

\section{Which Purine to Use?}

From our initial studies of the purine-loading phenomenon, the generalization emerged that organisms with high $(\mathrm{C}+\mathrm{G}) \%$ genomes would preferentially load with $\mathrm{G}$ residues, and organisms with low $(\mathrm{C}+\mathrm{G}) \%$ genomes would preferentially load with A residues (Bell and Forsdyke 1999b). This seemed logical, as organisms with a low $(\mathrm{C}+\mathrm{G}) \%$ would appear to have less flexibility for loading codons with scarce $G$ residues (e.g., the Gs would be required for critically placed codons, which might not match RNA loop regions). To our surprise, the present work (Table 1) indicates either that this generalization is not valid or that thermophiles are a special case. Perhaps because the strength of bonding between the $\mathrm{S}$ bases is greater than that between the $\mathrm{W}$ bases, it may be the avoidance of $\mathrm{C}$ residues as much as the inclusion of $G$ residues that generates such large Chargaff differences with respect to the $S$ bases in thermophiles. The unpaired Gs would locate to the loop regions of stem-loop secondary structures. In considering this matter, one should also take into account the fact that nearest neighbors are of considerable importance in base-pairing interactions (Turner 1996), and in low $(\mathrm{C}+\mathrm{G}) \%$ DNA an $\mathrm{S}$ base is more likely to have a $\mathrm{W}$ base nearest neighbor.

\section{METHODS}

\section{Chargaff Difference Analysis}

Chargaff's first parity rule for duplex DNA (\%A = \%T; $\% \mathrm{C}=\% \mathrm{G}$ ) applies, to a close approximation, to ssDNA (Chargaff's second parity rule; Chargaff 1979). Deviations from parity are referred to as Chargaff differences. The base composition of successive $1-\mathrm{kb}$ windows, moved in steps of $0.1 \mathrm{~kb}$, was assessed as described by Dang et al. (1998). Chargaff differences were either calculated as $(A-T) / W$ and $(C-G) / S$ and expressed as percentages or were expressed directly as positive or negative base excesses ( $-\mathrm{T}$, or $\mathrm{C}-\mathrm{G}$ ). Here, A, T, C, and $\mathrm{G}$ refer to the number of the corresponding base in a window. The direction of subtraction $(\mathrm{A}-\mathrm{T}$ or $\mathrm{T}-\mathrm{A})$ is determined alphabetically. $\mathrm{W}$ is the sum of the $\mathrm{W}$ bases $(\mathrm{A}+\mathrm{T})$ and $\mathrm{S}$ is the sum of the $S$ bases $(C+G)$.

\section{Purine-Loading}

Purine-loading of mRNAs is indicated when the regions of top DNA strands corresponding to leftward-transcribed ORFs are enriched in pyrimidines and when the regions of top DNA strands corresponding to rightward-transcribed ORFs are enriched in purines. These enrichments may be assessed at the DNA level as Chargaff differences. Because the direction of subtraction is determined alphabetically, purine-loading has been supported when Chargaff differences for the $\mathrm{W}$ bases $(A-T)$ are positive and/or Chargaff differences for the $S$ bases $(\mathrm{C}-\mathrm{G})$ are negative.

An overall index of the purine-loading of RNA was obtained by summing absolute Chargaff difference values (positive or negative base excesses per $1-\mathrm{kb}$ window) for the pyrimidine excess in leftward-transcribed ORFs and for the purine excess in rightward-transcribed ORFs. In circumstances where Szybalski's transcription direction rule was not followed, the corresponding absolute values were subtracted from the overall total.

When both purines contributed to purine-loading, the latter was assessed as the purine/pyrimidine ratio (Y/R; usually expressed as a percentage). In some circumstances codon choice provided a measure.

\section{Sequences}

Sequence information refers to the top strand as designated in the GenBank record. Genomic sequences examined were from Aquifex aeolicus (Deckert et al. 1998), A. fulgidus (Klenk et al. 1997), E. coli (Blattner et al. 1997), H. influenzae (Fleischmann et al. 1995), Methanobacterium thermoautotrophicum (Smith et al. 1997), M. jannaschii (Bult et al. 1996), Мycoplasma genitalium (Fraser et al. 1995), and Mycoplasma pneumoniae (Himmelreich et al. 1996). These sequences were analyzed with programs of the Genetics Computer Group (GCG, Madison, WI) and our own programs written as Unix scripts or in $\mathrm{C}++$. Codon usage tables for complete genomes, calculated using the GCG program CodonFrequency, were obtained from C. Brown (Department of Biochemistry, University of Otago, Dunedin, New Zealand).

\section{ACKNOWLEDGMENTS}

We thank Chris Brown for codon usage tables, Jim Gerlach for assistance with computer configuration, Gregory Hill for a 
program for determining optimum window sizes, and Robert Rasile for data on chloroplast genomes. The National Research Council of Canada, Academic Press, Cold Spring Harbor Laboratory Press, and Elsevier Publishing Corporation gave permission for the inclusion of full-text versions of the relevant papers cited herein at our internet site (http:// post.queensu.ca/ forsdyke/homepage.htm).

\section{REFERENCES}

Ball, L.A. 1973. Secondary structure and coding potential of the coat protein gene of bacteriophage MS2. Nature 310: 207-211.

Bell, S.J. and D.R. Forsdyke. 1999a. Accounting units in DNA. J. Theor. Biol. 197: 51-61.

- 1999b. Deviations from Chargaff's second parity rule correlate with direction of transcription. J. Theor. Biol. 197: 63-76.

Bell, S.J., Y.C. Chow, J.Y.K. Ho, and D.R. Forsdyke. 1998. Correlation of Chi orientation with transcription indicates a fundamental relationship between recombination and transcription. Gene 216: 285-292. [Correction 231: 213.]

Blattner, F.R., G. Plunkett III, C.A. Bloch, N.T. Perna, V. Burland, M. Riley, J. Collado-Vides, J.D. Glasner, C.K. Rode, G.F. Mayhew et al. 1997. The complete genome sequence of Escherichia coli K-12. Science 277: 1453-1474.

Bronson, E.C. and J.N. Anderson. 1994. Nucleotide composition as a driving force in the evolution of retroviruses. J. Mol. Evol. 38: $506-532$.

Bult, C.J., O. White, G.J. Olsen, L. Zhou, R.D. Fleischmann, G.G. Sutton, J.A. Blake, L.M. FitzGerald, R.A. Clayton, J.D. Gocayne et al. 1996. Complete genomic sequence of the methanogenic archaeon, Methanococcus jannaschii. Science 273: 1058-1072.

Cantor, C.R. and P.R. Schimmel. 1980. Statistical mechanics and kinetics of nucleic acid interactions. In Biophysical Chemistry, pp. 1183-1264. Freeman, San Francisco, CA.

Chargaff, E. 1979. How genetics got a chemical education. Ann. N.Y. Acad. Sci. 325: 345-360.

Cristillo, A.R. 1998. "Characterization of $\mathrm{G}_{0} / \mathrm{G}_{1}$ switch genes in cultured T lymphocytes". Ph.D. thesis. Queen's University, Kingston, Ontario Canada.

Dang, K.D., P.B. Dutt, and D.R. Forsdyke. 1998. Chargaff difference analysis of the bithorax complex of Drosophila melanogaster. Biochem. Cell Biol. 76: 129-137.

Deckert, G., P.V. Warren, T. Gaasterland, W.G. Yong, A.L. Lenox, D.E. Graham, R. Overbeek, M.A. Snead, M. Keller, M. Aujay et al. 1998. The complete genome of the hyperthermophilic bacterium Aquifex aeolicus. Nature 392: 353-358.

Eguchi, Y., T. Itoh, and J. Tomizawa. 1991. Antisense RNA. Аnnu. Rev. Biochem. 60: 631-652.

Fire, A. 1999. RNA-triggered gene silencing. Trends Genet. 15: $358-363$.

Fleischmann, R.D., M.D. Adams, O. White, R.A. Clayton, E.F. Kirkness, A.R. Kerlavage, C.J. Bult, J.F. Tomb, B.A. Dougherty, J.M. Merrick et al. 1995. Whole-genome random sequencing and assembly of Haemophilus influenzae Rd. Science 269: 496-512.

Forsdyke, D.R. 1995. Entropy-driven protein self-aggregation as the basis for self/not-self discrimination in the crowded cytosol. J. Biol. Syst. 3: 273-287.

. 1996. Different biological species "broadcast" their DNAs at different $(\mathrm{C}+\mathrm{G}) \%$ "wavelengths." J. Theor. Biol. 178: 405-417.

- 1998. An alternative way of thinking about stem-loops in

DNA. A case study of the GOS2 gene. J. Theor. Biol. 192: 489-504. . 1999a. Heat shock proteins as mediators of aggregation-induced "danger" signals: Implications of the slow evolutionary fine-tuning of sequences for the antigenicity of cancer cells. Cell Stress Chaperones 4: 205-210.

- 1999b. The origin of species revisited. Queen's Q.

106: $112-133$.

. 1999c. Two levels of information in DNA. Relationship of Romanes' "intrinsic" variability of the reproductive system, and Bateson's "residue", to the species-dependent component of the base composition, $(\mathrm{C}+\mathrm{G}) \%$. J. Theor. Biol. 201: 47-61.

Fraser, C.M., J.D. Gocayne, O. White, M.D. Adams, R.A. Clayton, R.D. Fleischmann, C.J. Bult, A.R. Kerlavage, G. Sutton, J.M. Kelley et al. 1995. The minimal gene complement of Mycoplasma genitalium. Science 270: 397-403.

Fulton, A.B. 1982. How crowded is the cytoplasm? Cell 30: $345-347$.

Grantham, R. 1980. Workings of the genetic code. Trends Biochem. Sci. 5: 327-331.

Himmelreich, R., H. Hilbert, H. Plagens, E. Pirkl, B.-C. Li, and R. Herrmann. 1996. Complete sequence analysis of the genome of the bacterium Mycoplasma pneumoniae. Nucleic Acids Res. 24: $4420-4449$.

Jaenicke, R. and G. Bohm. 1998. The stability of proteins in extreme environments. Curr. Opin. Struct. Biol. 8: 738-748.

Kagawa, Y., H. Nojima, N. Nukiwa, M. Ishizuka, T. Nakajima, T. Yasuhara, T. Tanaka, and T. Oshima. 1984. High guanine plus cytosine content in the third letter of codons of an extreme thermophile. J. Biol. Chem. 259: 2956-2960.

Klenk, H.-P., R.A. Clayton, J.F. Tomb, O. White, K.E. Nelson, K.A. Ketchum, R.J. Dodson, M. Gwinn, E.K. Hickey, J.D. Peterson et al. 1997. The complete genome sequence of the hyperthermophilic sulphate-reducing archaeon Archaeoglobus fulgidus. Nature 390: 364-370.

Lauffer, M.A. 1975. Entropy-driven processes in biology. Springer-Verlag, New York, NY.

Lee, M.-A., M.E. Diamond, and J.L. Yates. 1999. Genetic evidence that EBNA-1 is needed for efficient stable latent infection by Epstein-Barr virus. J. Virol. 73: 2974-2982.

Lobry, J.R. 1996. Origin of replication of Mycoplasma genitalium. Science 272: 745-746.

Romanes, G.J. 1886. Physiological selection: an additional suggestion on the origin of species. J. Linn. Soc. (Zool.) 19: 337-411.

Smith, D.R., L.A. Doucette-Stamm, C. Deloughery, H. Lee, J. Dubois, T. Aldredge, R. Bashirzadeh, D. Blakely, R. Cook, K. Gilbert et al. 1997. Complete genome sequence of Methanobacterium thermoautotrophicum $\Delta \mathrm{H}$ : Functional analysis and comparative genomics. J. Bacteriol. 179: 7135-7155.

Smithies, O., W.R. Engels, J.R. Devereux, J.L. Slightom, and S.-H Chen. 1981. Base substitutions, length differences and DNA strand asymmetries in the human ${ }^{\mathrm{G}} \gamma$ and ${ }^{\mathrm{A}} \gamma$ fetal globin gene region. Cell 26: 345-353.

Sueoka, N. 1961. Compositional correlation between deoxyribonucleic acid and protein. Cold Spring Harbor Symp. Quant. Biol. 26: 35-43.

Szybalski, W., H. Kubinski, and O. Sheldrick. 1966. Pyrimidine clusters on the transcribing strand of DNA and their possible role in the initiation of RNA synthesis. Cold Spring. Harbor Symp. Quant. Biol. 31: 123-127.

Turner, D.H. 1996. Thermodynamics of base pairing. Curr. Opin. Struct. Biol. 6: 299-304.

Zuckerkandl, E. 1986. Polite DNA: Functional density and functional compatibility in genomes. J. Mol. Evol. 24: 12-27.

Received August 23, 1999; accepted in revised form December 16, 1999. 


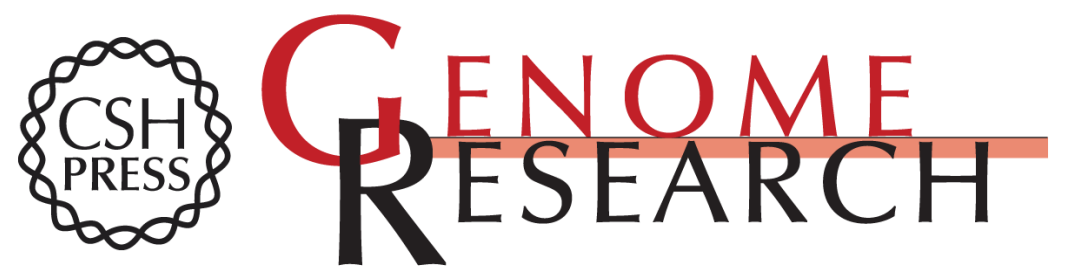

\title{
Thermophilic Bacteria Strictly Obey Szybalski's Transcription Direction Rule and Politely Purine-Load RNAs with Both Adenine and Guanine
}

\author{
Perry J. Lao and Donald R. Forsdyke
}

Genome Res. 2000 10: 228-236

Access the most recent version at doi:10.1101/gr.10.2.228

References This article cites 32 articles, 10 of which can be accessed free at:

http://genome.cshlp.org/content/10/2/228.full.html\#ref-list-1

\section{License}
Email Alerting Receive free email alerts when new articles cite this article - sign up in the box at the Service top right corner of the article or click here.

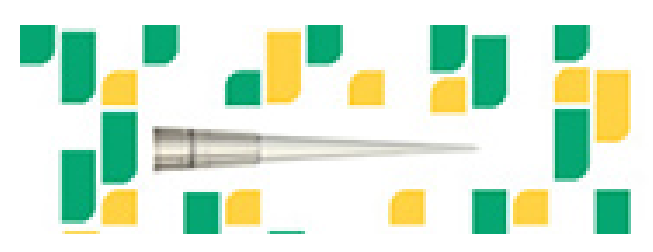

